\title{
In vitro activity of biapenem and comparators against multidrug-resistant and carbapenem-resistant Acinetobacter baumannii isolated from tertiary care hospitals in Thailand
}

\author{
Jantana Houngsaitong ${ }^{1}$, \\ Preecha Montakantikul', \\ Taniya Paiboonwong ${ }^{1}$, \\ Mullika Chomnawang ${ }^{2}$, \\ Piyatip Khuntayaporn ${ }^{2}$, \\ Suvatna Chulavatnatol ${ }^{1 *}$ \\ ${ }^{1}$ Department of Pharmacy, Faculty of Pharmacy, \\ Mahidol University, Bangkok, Thailand \\ ${ }^{2}$ Department of Microbiology, Faculty of \\ Pharmacy, Mahidol University, Bangkok, \\ Thailand
}

*Corresponding author:

Suvatna Chulavatnatol

suvatna.chu@mahidol.ac.th

\section{KEYWORDS:}

Biapenem; Multidrug-resistant $A$.

Baumannii; Carbapenem-resistant

A. Baumannii; Thailand

https://www.pharmacy.mahidol.ac.th/journal/ (C) Faculty of Pharmacy, Mahidol University (Thailand) 2020

\begin{abstract}
Acinetobacter baumannii is one of nosocomial pathogen which emerges as multidrug-resistance worldwide. Multidrugresistant $A$. baumannii (MDRAB) and carbapenem-resistant A. baumannii (CRAB) are highly concerned due to limitation of therapeutic options. Antibacterial activity of biapenem was explored in order to overcome bacterial resistance. A total of 412 A. baumannii clinical isolates from 13 tertiary care hospitals in Thailand were collected. MIC values of biapenem and comparators; imipenem, meropenem, colistin, sulbactam, ciprofloxacin ceftazidime and fosfomycin sodium, were determined by broth microdilution method in accordance with the Clinical and Laboratory Standards Institute (CLSI) guidelines (2016). In total, 320 isolates (77.67\%) were MDRAB and 328 isolates (79.61\%) were CRAB while 58 isolates $(14.07 \%)$ were colistin-resistant AB. A. baumannii showed widespread resistance to ceftazidime, ciprofloxacin and carbapenems in more than $90 \%$ of the strains; resistance to sulbactam, fosfomycin, and colistin were $85 \%, 60 \%$, and $15 \%$ respectively. By comparison among carbapenems, biapenem showed $\mathrm{MIC}_{50 / 90}$ of 16/32 which were at least 2 folds lower than imipenem and meropenem $\left(\mathrm{MIC}_{50 / 90} ; 32 / 128,32 / 64\right.$, respectively). In addition, $15 \%$ of imipenem and meropenemresistant $A$. baumannii were susceptible to biapenem. Also, 19\% of colistin-resistant $A$. baumannii were susceptible to biapenem. Although more than $90 \%$ of MDRAB and CRAB were resistant to carbapenems, biapenem showed a good activity over other carbapenems. This drug might be a therapeutic option mono or combination therapy besides other antimicrobial agents.
\end{abstract}

\section{INTRODUCTION}

The emerging problem of antibiotic resistance, especially among Gram-negative bacteria (GNB) in nosocomial infection, is a major global concern. The majority of Gramnegative infections in intensive care unit (ICU) are caused by extended spectrumbeta-lactamase(ESBL) Enterobacteriaceae, Pseudomanas aeruginosa, and Acinetobacter baumanii ${ }^{1}$. The increasing of Acinetobacter spp. resistance could be an alarming issue. Moreover, 
A. baumannii has emerged as multidrug-resistant A. baumannii (MDRAB), carbapenem-resistant A. baumannii (CRAB), and colistin-resistant strains (CoRAB) which are associated with high mortality rates and longer hospital stays ${ }^{2}$. Carbapenems and colistin have been commonly used as the treatment of choice for MDRAB infections ${ }^{3}$. However, nephrotoxicity and neurotoxicity from colistin use have been reported ${ }^{4-5}$; while, MDRAB isolates which are resistant to carbapenems and colistin have been increasingly reported worldwide ${ }^{6-7}$.

Biapenem is a new parenteral carbapenem antibiotic, which are inhibit bacterial cell wall synthesis, and has a wide range of antibacterial activity encompassing many gram-negative and gram-positive aerobic and anaerobic bacteria, including species producing $\beta$-lactamases. Biapenem has a penicillin-like five-membered ring, but the sulfur at C-1 in the five-membered ring is replaced with a carbon atom and a double bond between $\mathrm{C}-2$ and $\mathrm{C}-3$ is introduced. Moreover, unlike imipenem, biapenem has a $1 \beta$-methyl group at the $\mathrm{C} 1$ position which is stable to hydrolysis by human renal dihydropeptidase-1 (DHP-1) and it does not require the co-administration of a DHP-1 inhibitor ${ }^{8}$. To counter the increasing prevalence of MDR-AB, biapenem, the latest broad-spectrum carbapenem approved in several countries, is more stable against carbapenemase than other carbapenems 9 . The data from Siriraj Hospital, Thailand showed the susceptibility of biapenem against extended spectrum beta-lactamase (ESBL)-producing E. coli, ESBL-producing $K$. pneumoniae, $P$. aeruginosa and $A$. baumannii were $100 \%, 100 \%, 70 \%$, and $37 \%$ respectively, during 2006 to $2009^{10}$. Similar to other studies in China ${ }^{11}$ and India ${ }^{12}$, biapenem showed good activity against ESBL-producing E. coli, K. pneumoniae, $P$. aeruginosa, and $A$. baumanii. Thus, biapenem has emerged as a new treatment option for MDRAB infections. Nonetheless, none of prevalence studies which collected large number of $A$. baumanii isolates in tertiary care hospital in Thailand showed susceptibility patterns of biapenem and other antimicrobial agents against these organisms. In this study, we determined the in vitro activity of biapenem against $A$. baumannii causing serious infections in hospitalised patients from tertiary care hospitals in Thailand.

\subsection{MATERIALS AND METHODS}

\subsection{Study centers and bacterial isolates}

A. baumannii clinical isolates were collected from patients admitted to 13 tertiary care hospitals in Thailand in 2017. These 13 tertiary care hospitals were 4 hospitals from central region including Bangkok, 3 hospitals fromnorthern region, 3 hospitals from north-eastern region, 2 hospitals from southern region, and 1 hospital from eastern region of the country. Non-duplicate consecutive isolates from distinct infectious episodes were obtained from admitted patients. All study isolates were sent to microbiology laboratory (Department of Microbiology, Faculty of Pharmacy, Mahidol University, Bangkok, Thailand).

This study protocol was approved by The Ethics Committee of Faculty of Dentistry/Faculty of Pharmacy, Mahidol University (COA.No.MUDT/PY-IRB 2017/040.2607).

\subsection{Antimicrobial susceptibility testing and interpretation}

Antimicrobial susceptibility testing was performed by broth microdilution in cation-adjusted Mueller-Hinton broth (CAMHB) according to Clinical Laboratory Standard Institute (CLSI) guidelines $2016^{13}$. A. baumannii isolates were tested against biapenem and comparators; imipenem, meropenem, colistin, sulbactam, ciprofloxacin, ceftazidime and fosfomycin sodium. The concentration ranges tested in two-fold dilutions were carbapenems $(0.125-1024 \mu \mathrm{g} / \mathrm{mL})$, colistin $(0.0625-512 \mu \mathrm{g} / \mathrm{mL})$, sulbactam $(0.5-2048 \mu \mathrm{g} /$ $\mathrm{mL})$, ciprofloxacin $(2-512 \mu \mathrm{g} / \mathrm{mL})$, ceftazidime $(0.125-512 \mu \mathrm{g} / \mathrm{mL})$ and fosfomycin sodium $(8-4096 \mu \mathrm{g} / \mathrm{mL})$. The reference isolates $E$. coli ATCC 25922 were used as positive quality control isolates.

MICs and susceptibility rates were interpreted according to the CLSI guidelines $(2016)^{13}$. No breakpoints for fosfomycin sodium are available from the CLSI guidelines. Thus, the CLSI guidelines for Enterobacteriaceae were used for fosfomycin sodium (susceptibility, $\leq 64 \mu \mathrm{g} / \mathrm{mL}$; resistance, $\geq 256 \mu \mathrm{g} / \mathrm{mL})^{13}$. Additionally, they were divided into the CRAB and MDR groups according to the antimicrobial susceptibility patterns as the following antimicrobial categories: antipseudomonal carbapenems, antipseudomonal fluoroquinolones, 
$\beta$-lactamase inhibitors, extended-spectrum cephalosporins, and polymyxins. CRAB was defined as acquired nonsusceptible (intermediate or resistant) to at least one carbapenem (excluding ertapenem). MDRAB was defined as non-susceptible to multiple antibiotics, often defined as three or more antimicrobials (e.g. aminoglycoside, ampicillin-sulbactam, antipseudomonal carbapenem, antipseudomonal cephalosporin, and fluoroquinolone) ${ }^{14}$.

\subsection{Antimicrobial agents}

Most antimicrobial agent standard powder including imipenem and cilastatin sodium, meropenem, ciprofloxacin, ceftazidime, sulbactam, colistin sulphate were purchased from Tokyo Chemical Industry, Japan. Biapenem and fosfomycin standard powder were supported by Thai Meiji Pharmaceutical Co., Ltd, Thailand.

\subsection{Data analysis}

All relevant data including the proportion of resistance pattern, susceptibility of each antimicrobial agent, the MIC range, $\mathrm{MIC}_{50 / 90}$ were performed by using the latest version of the MS Excel 2016. Data were expressed as range, percentage, and mean \pm SD.

\section{RESULTS}

\subsection{Study isolates}

A total of 412 A. baumannii clinical isolates were submitted to in vitro activity testing. All of the isolates were derived from patients with hospital-acquired infections. Three-hundred and twenty-one (77.9\%), 37 (8.98\%), 23 (5.58\%), $19(4.61 \%)$, and $12(2.91 \%)$ of the isolates were obtained from sputum, pus, blood, urine, and other specimens, respectively. Escherichia coli ATCC 25922 and Pseudomonas aeruginosa ATCC 27853 were used as quality control (QC) organisms to ensure proper test conditions and procedures for susceptibility testing. The validation of QC results was based on breakpoints in the CLSI recommendations. The MICs of QC strains produced $99.5 \%$ within established ranges.

\subsection{Susceptibility to antimicrobial agents}

According to MIC results from the reference
BMD method, 412 non-duplicate clinical isolates of $A$. baumannii isolates were classified as 76 non-MDR (18.45\%), 320 MDRAB (77.67\%), 328 CRAB (79.61\%), and 58 CoRAB (14.07\%). The antibacterial MIC range, $\mathrm{MIC}_{50}$ and $\mathrm{MIC}_{90}$ of MDR and CRAB isolates were 4-128 fold higher than non-MDR isolates. Among the non-MDR isolates, the most isolates ( $\geq 90 \%$ ) had MICs of imipenem, meropenem, and biapenem $\leq 2 \mu \mathrm{g} / \mathrm{mL}$ and $\mathrm{MIC}_{90} \leq 2 \mu \mathrm{g} / \mathrm{mL}$, whilst only $2.6 \%$ of imipenem to non-MDR isolates had MIC $>4 \mu \mathrm{g} / \mathrm{mL}$. On the contrary, the most isolates $(>80 \%)$ had MICs of meropenem, and biapenem $\geq 16 \mu \mathrm{g} / \mathrm{mL}$ and $\mathrm{MIC}_{90}$ of carbapenems was $\geq 32 \mu \mathrm{g} / \mathrm{mL}, \geq 64 \mu \mathrm{g} / \mathrm{mL}$ in MDRAB/CRAB, CoRAB, respectively. Therefore, the MDR A. baumannii population had significantly higher MICs and more non-susceptible isolates than the non-MDR isolates. When considered in geographic part and source of specimen, the MIC range, $\mathrm{MIC}_{50 / 90}$ reported no more than 2-fold dilution when compared with the overall mean.

Over $95 \%$ of MDRAB and CRAB isolates exhibited high resistance to cephalosporins, ciprofloxacin, and carbapenems including imipenem, meropenem except biapenem (92\%). Whereas, colistin was found to be the most effective agent which showed only $14 \%$ resistance.

Totally, 328 A. baumannii clinical isolates were identified as CRAB, with the MICs of carbapenems ranging from 4 to $1024 \mu \mathrm{g} / \mathrm{mL}$. The rate of carbapenem resistance was more than $70 \%$ while, all carbapenem-resistant isolates had $\mathrm{MIC}_{90}$ to imipenem, meropenem $>64 \mu \mathrm{g} / \mathrm{mL}$ and biapenem $32 \mu \mathrm{g} / \mathrm{mL}$. In addition, 320 A.baumannii clinical isolates were identified as MDRAB, with $>90 \%$ of isolates resistant to 3 groups of antimicrobials which were carbapenem, cephalosporins and fluoroquinolones. On the other hand, the rate of colistin resistance was $15 \%$ in $\mathrm{CRAB}$ and MDRAB while, the $\mathrm{MIC}_{50}$ and $\mathrm{MIC}_{90}$ were 0.5 and $4 \mu \mathrm{g} / \mathrm{mL}$, respectively. For colistin testing, $85.9 \%$ of $A$. baumannii isolates were classified as susceptible to colistin. Although MDRAB had a broader range of MICs than non-MDR isolates $(512-0.0625 \mu \mathrm{g} / \mathrm{mL}$ vs. $4-0.031 \mu \mathrm{g} / \mathrm{mL}$, respectively), the $\mathrm{MIC}_{50}$ and $\mathrm{MIC}_{90}$ values of colistin for both groups were not different. Among CoRAB isolates, high rate of resistance $(>80 \%)$ were found in most antimicrobial agents, but only fosfomycin showed $79.14 \%$ of 
resistance. MIC range, $\mathrm{MIC}_{50 / 90}$ and percent susceptibility rates for $A$. baumannii isolates were summarized in Table 1. The cumulative
MIC distributions of antimicrobial tested against MDRAB, CRAB and CoRAB were shown in Figure 1.

Table 1. MIC values for individual antimicrobial agents of AB, non-MDR, MDRAB, CRAB, and Colistin-resistant $\mathrm{AB}$

\begin{tabular}{|c|c|c|c|c|c|c|c|c|}
\hline \multirow{2}{*}{ Type of organisms } & \multicolumn{8}{|c|}{ Antimicrobial agents } \\
\hline & Imipenem & Meropenem & Biapenem & Colistin & Sulbactam & Ceftazidime & Ciprofloxacin & Fosfomycin \\
\hline $\begin{array}{l}\text { MDRAB }(\mathbf{N}=\mathbf{3 2 0}) \\
-\% \text { susceptible } \\
-\mathrm{MIC}_{50} \\
-\mathrm{MIC}_{90} \\
\text { - MIC range }\end{array}$ & $\begin{array}{l}2.81 \\
32 \\
128 \\
0.25-1024\end{array}$ & $\begin{array}{l}3.13 \\
32 \\
64 \\
0.125-256\end{array}$ & $\begin{array}{l}7.92 \\
16 \\
32 \\
0.125-256\end{array}$ & \begin{tabular}{|l}
84.68 \\
0.5 \\
4 \\
$0.063-512$
\end{tabular} & $\begin{array}{l}7.62 \\
32 \\
128 \\
1-2048\end{array}$ & $\begin{array}{l}0 \\
>512 \\
>512 \\
16->512\end{array}$ & $\begin{array}{l}0.94 \\
64 \\
>256 \\
0.5->512\end{array}$ & \begin{tabular}{|l}
40 \\
256 \\
1024 \\
$32->2048$
\end{tabular} \\
\hline $\begin{array}{l}\text { CRAB }(\mathbf{N}=\mathbf{3 2 8}) \\
-\% \text { susceptible } \\
-\mathrm{MIC}_{50} \\
-\mathrm{MIC}_{90} \\
\text { - MIC range } \\
\end{array}$ & $\begin{array}{l}- \\
32 \\
128 \\
4-1024 \\
\end{array}$ & $\begin{array}{l}1.52 \\
32 \\
64 \\
0.5-256 \\
\end{array}$ & $\begin{array}{l}8.23 \\
16 \\
32 \\
0.125-256 \\
\end{array}$ & \begin{tabular}{|l}
85.06 \\
0.5 \\
4 \\
$0.063-512$ \\
\end{tabular} & $\begin{array}{l}11.89 \\
32 \\
128 \\
0.5-2048 \\
\end{array}$ & \begin{tabular}{|l}
2.74 \\
$>512$ \\
$>512$ \\
$2->512$ \\
\end{tabular} & \begin{tabular}{|l}
3.35 \\
64 \\
256 \\
$0.125->512$ \\
\end{tabular} & \begin{tabular}{|l}
40.85 \\
256 \\
1024 \\
$32->2048$ \\
\end{tabular} \\
\hline $\begin{array}{l}\text { CoRAB }(\mathbf{N}=\mathbf{5 8}) \\
-\% \text { susceptible } \\
-\mathrm{MIC}_{50} \\
-\mathrm{MIC}_{90} \\
\text { - MIC range } \\
\end{array}$ & $\begin{array}{l}10.34 \\
32 \\
128 \\
0.25-1024\end{array}$ & $\begin{array}{l}17.24 \\
32 \\
128 \\
0.25-256 \\
\end{array}$ & $\begin{array}{l}17.24 \\
16 \\
32 \\
0.125-256 \\
\end{array}$ & \begin{tabular}{|l}
0 \\
4 \\
16 \\
$4-512$ \\
\end{tabular} & $\begin{array}{l}17.24 \\
32 \\
128 \\
0.5-2048 \\
\end{array}$ & $\begin{array}{l}10.34 \\
>512 \\
>512 \\
4->512 \\
\end{array}$ & \begin{tabular}{|l}
10.34 \\
64 \\
$>256$ \\
$0.5->512$ \\
\end{tabular} & \begin{tabular}{|l}
20.68 \\
256 \\
1024 \\
$64->2048$ \\
\end{tabular} \\
\hline $\begin{array}{l}\text { Non- MDRAB } \\
(\mathbf{N}=\mathbf{7 6}) \\
-\% \text { susceptible } \\
-\mathrm{MIC}_{50} \\
-\mathrm{MIC}_{90} \\
\text { - MIC range }\end{array}$ & $\begin{array}{l}94.74 \\
0.5 \\
2 \\
0.125-16\end{array}$ & $\begin{array}{l}96.05 \\
0.5 \\
1 \\
0.063-4\end{array}$ & $\begin{array}{l}97.37 \\
0.125 \\
0.25 \\
0.015-4\end{array}$ & $\begin{array}{l}93.42 \\
0.25 \\
2 \\
0.03-4\end{array}$ & $\begin{array}{l}85.52 \\
2 \\
32 \\
0.5-512\end{array}$ & \begin{tabular}{|l}
76.32 \\
8 \\
512 \\
$2->512$
\end{tabular} & $\begin{array}{l}78.94 \\
0.5 \\
8 \\
0.125-128\end{array}$ & \begin{tabular}{|l}
48.68 \\
128 \\
512 \\
$8-1024$
\end{tabular} \\
\hline $\begin{array}{l}\text { Total }(\mathbf{N}=\mathbf{4 1 2}) \\
-\% \text { susceptible } \\
-\mathrm{MIC}_{50} \\
-\mathrm{MIC}_{90} \\
\text { - MIC range }\end{array}$ & $\begin{array}{l}19.67 \\
32 \\
64 \\
0.125-1024\end{array}$ & $\begin{array}{l}20.87 \\
32 \\
64 \\
0.063-256\end{array}$ & $\begin{array}{l}26.21 \\
8 \\
32 \\
0.016-256\end{array}$ & $\begin{array}{l}85.92 \\
0.5 \\
4 \\
0.03-512\end{array}$ & $\begin{array}{l}31.55 \\
16 \\
128 \\
0.5-2048\end{array}$ & $\begin{array}{l}18.20 \\
>512 \\
>512 \\
2->512\end{array}$ & $\begin{array}{l}17.96 \\
64 \\
256 \\
0.125->512\end{array}$ & $\begin{array}{l}40.78 \\
256 \\
1024 \\
8->2048\end{array}$ \\
\hline
\end{tabular}


(a)

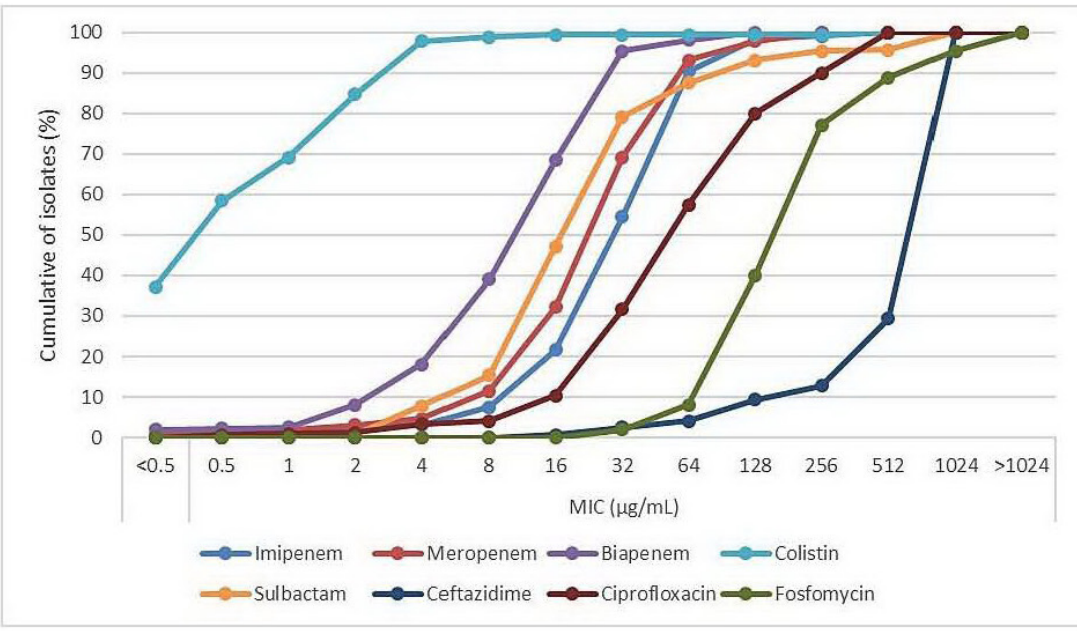

(b)

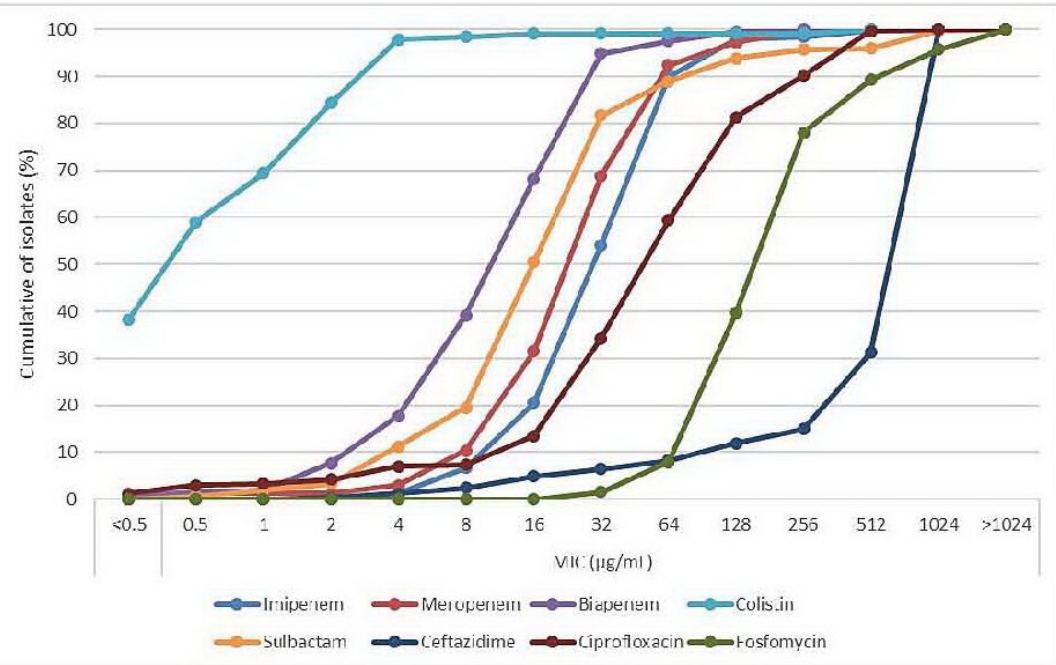

(c)

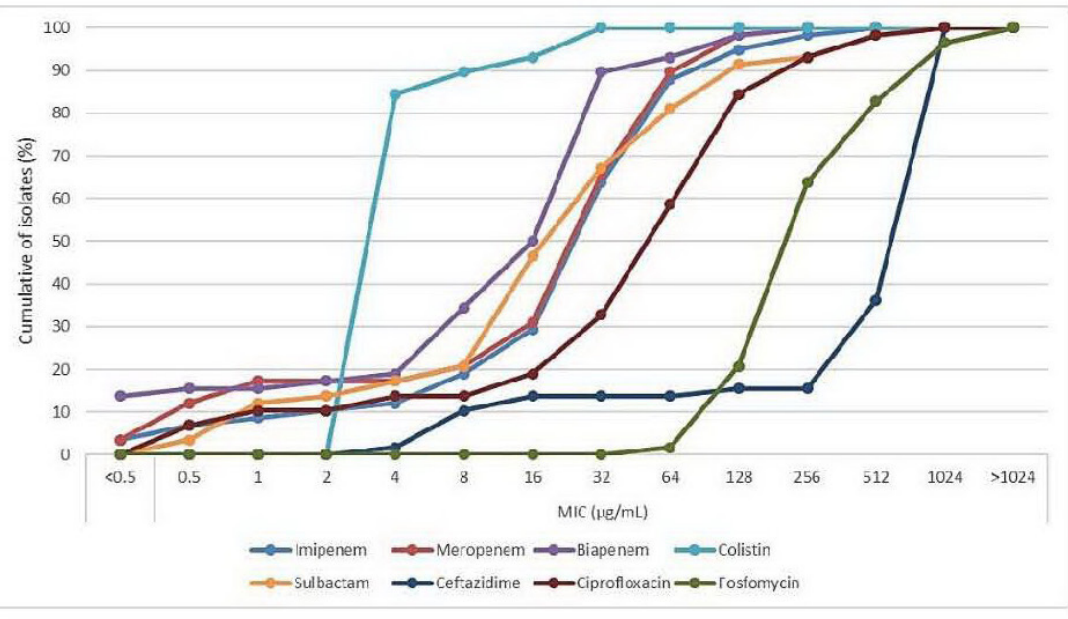

Figure 1. Cumulative minimum inhibitory concentration (MIC) distribution for all antimicrobial tested against (a) MDRAB, (b) CRAB and (c) Colistin-resistant AB 
Biapenem has higher activity against $A$. baumannii when compared with other carbapenems, as shown in this study, with an overall $\mathrm{MIC}_{90}$ of $32 \mu \mathrm{g} / \mathrm{mL}$ for biapenem amongst MDR, CRAB, and CoRAB isolates which was lower than that of imipenem and meropenem at least 2 to 4 -fold dilution. Moreover, $\geq 15 \%$ of isolates which were resistant to imipenem and/or meropenem showed susceptible to biapenem.

\section{DISCUSSION}

The prevalence of antibiotic-resistant pathogens such as $A$. baumannii is increasing worldwide and specifically in the Asia-Pacific region ${ }^{15-16}$. This dilemma is complicated by limited available antimicrobial choices and the recognition of problems with antimicrobial use. In present study, from 2016 till 2017, a significant increase of multidrug-resistant and carbapenem-resistant A. baumannii in a tertiary care hospital in Thailand during 2016 till 2017 was observed. Carbapenem resistance rates were high for MDRAB and CRAB, in which imipenem and meropenem were less active than biapenem. Based on $\mathrm{MIC}_{90}$ values, biapenem was 2-fold and 4-fold more active against MDRAB and $\mathrm{CRAB}$ than imipenem and meropenem respectively. Most of A. baumannii remained highly susceptible to colistin. These results are consistent with the prevalence of CRAB and MDRAB in Southeast Asia ${ }^{15-16}$.

Result from the Comparative Activity of Carbapenems Testing (COMPACT) Asia-Pacific Study during the latter half of 2008 and early 2009, showed MICs for carbapenems including doripenem, meropenem and imipenem against A. baumannii of $>64 \mu \mathrm{g} / \mathrm{mL}$ for both MIC50 and MIC90. Susceptibility rates for A. baumannii were approximately $20-30 \%$ for all carbapenems tested ${ }^{17}$. Similarly, the COMPACT II Asia-Pacific Study during April-July 2010 showed $73 \%$ of A. baumannii not susceptible to carbapenems and $\mathrm{MIC}_{50 / 90}$ were $32 />64 \mu \mathrm{g} / \mathrm{mL}^{18}$. Data according to the latest NARST report, over $70 \%$ of isolates tested (42,212 isolates) between January and December 2018 were found to be resistant to carbapenem, ampicillin/sulbactam, and piperacillin/ tazobactam which was a significant increase from $58.2 \%$ (13,645 isolates) in 2010 $0^{19-20}$.

Among the eight antibiotics tested, MDRAB and $C R A B$ showed widespread resistance to ceftazidime, ciprofloxacin and carbapenems in more than $90 \%$ of the strains. The reason of high resistance rate could be explained by two factors which were the pathogenic organisms, and the host status. The first reason was that the pathogens were obtained from tertiary care hospitals in Thailand, where risk of drug resistance organisms was high. In contrast to the NARST report ${ }^{19-20}$, specimens were collected from various types of hospitals. The cause of high resistance rates of infection in tertiary care hospitals was finally derived from several factors including the inappropriateness of antibiotic use. In particular, prior antibiotic use, such as carbapenems, was an important factor that contributed to the selection of such organisms ${ }^{21}$. The second reason was patients admitted in tertiary care hospitals were most likely had high risk of A. baumannii resistance because physiological defense barriers were interrupted by several treatment modalities. Consequently, patients might suffer from infections caused by MDR pathogens. These conditions of the patient generally require broad spectrum antibiotics, and thus lead to highly resistance pattern. In addition, there were other precipitating factors such as ventilator support, urinary and/or intravenous catheter. These all increased the risk of colonization or infection with $A$. baumannii ${ }^{22}$.

However, more than $15 \%$ of imipenem and meropenem resistant strains were sensitive to biapenem. Several explanations include structure relationship of biapenem and the different resistance mechanisms. The bla $a_{O X A-23}$ was reported as the OXAtype carbapenemase-encoding genes in Thailand. Biapenem has good activity against $A$. baumannii which expresses the OXA-type carbapenemase. Biapenem affects to class B, D carbapenemase enzyme in several ways. These are binding carbepenemase residue structures which reveal the conformational change at the active site, strong hydrogen bond with composition of NDM-1 which affects on biapenem binding and hydrolysis against Gram negative bacteria .

From this in vitro data, it can be proposed that biapenem might have role in the treatment of MDRAB, CRAB, and CoRAB in two means which are increasing dose of biapenem and/ or use as combination with other antimicrobial agents. Available pharmacokinetic/pharmacodynamic data 
on biapenem, the finding of a significant correlation between drug concentrations and the probability of attaining pharmacodynamic target (PTA) in various populations showed $>75 \%$ PTA values for achieving $\geq 40 \% \mathrm{~T}>\mathrm{MIC}$ at MIC $4 \mu \mathrm{g} / \mathrm{mL}$ for doses of standard regimen and extended infusion (3-h infusion). With only a dose of $300 \mathrm{mg}$ q8h (3-h infusion) and $600 \mathrm{mg} \mathrm{q} 8 \mathrm{~h}$ (3-h infusion) could be achieved PTA $>75 \%$ for $\geq 40 \% \mathrm{~T}>\mathrm{MIC}$ at MIC $8 \mu \mathrm{g} / \mathrm{mL}$. However, no regimens achieved PTA $\geq 80 \%$ at MIC $32 \mu \mathrm{g} / \mathrm{mL}$, which was $\mathrm{MIC}_{90}$ of this study ${ }^{23}$. Thus, further investigation on increased dose of biapenem might be needed. Additionally, the role of biapenem in combination with other antimicrobial agents might be possible. The findings of this study showed higher susceptibility to fosfomycin, sulbactam and colistin for both MDRAB and CRAB than other antimicrobials. The synergistic or additive effect of carbapenem with fosfomycin, sulbactam and colistin were reported against $A$. baumannii ${ }^{24-25}$. Therefore, the rates of synergy and characterise of biapenem in combination with colistin, fosfomycin, and sulbactam against carbapenem-resistant $A$. baumannii should be further studied utilizing checkerboard and time-kill experiments.

The strengths of this study lie in the large number of isolates from several tertiary-care hospitals across Thailand, and probably attributable to the infection control and antimicrobial overuse in place in each center. However, this study still has several limitations that should be noted. First, the study was not able to evaluate the information regarding antimicrobial administration, which could be one of the risk factors for MDRAB and CRAB acquisition. Second, some antimicrobials were not tested in this study which were amikacin and tigecycline. Last genotypic mechanism could not be detected.

\section{CONCLUSIONS}

A. baumannii resistance seems to be a complex phenomenon, in which isolates of different sources and different resistance patterns. Our data supported that, colistin was found to be better against MDRAB and CRAB compared to other antimicrobial agents. However, prudent use of colistin is needed to avoid an increase in colistinresistance mechanisms. While biapenem showed a good activity over other carbapenems against the MDRAB, CRAB and CoRAB. Accordingly, this drug might be a therapeutic option which might be used as high dose monotherapy or in combination for highly resistant bacteria. Further studies are needed to figure out the appropriate dose of biapenem and role of combination therapy with other antimicrobials against $A$. baumannii resistance strains.

\section{ACKNOWLEDGEMENTS}

We thank all staff in hospitals for collecting the clinical isolates for this work; Buddhachinaraj Phitsanulok Hospital, Buriram Hospital, Chonburi Hospital, Klang Hospital, Maharaj Nakorn Chiang Mai Hospital, Sawanpracharak Hospital, Maharaj Nakhon Si Thammarat Hospital, Nakornpathom Hospital, Samutsakhon Hospital, Suppasitthiprasong Hospital, Suratthani Hospital, Surin Hospital, and Taksin Hospital. We are also very grateful to Thai Meiji Co.,Ltd., Thailand for supporting the standard powder of biapenem and fosfomycin.

\section{Conflict of interest (If any)}

This study protocol was principally initiated by all investigators. Biapenem and fosfomycin standard powder were supported by Thai Meiji Co.,Ltd., Thailand. The company had no role in protocol development, data collection, data analysis, results, discussion and conclusion of this work. The authors had no conflict of interest in this work.

\section{Funding}

None to declare

\section{Ethical approval}

This study was approved by The Ethics Committee of Faculty of Dentistry/Faculty of Pharmacy, Mahidol University (COA.No.MU-DT/PY-IRB 2017/040.2607).

\section{Article info:}

Received August 8, 2019

Received in revised form December 13, 2019

Accepted December 28, 2019

\section{REFERENCES}

1. MacVane SH. Antimicrobial resistance in the intensive care unit: a focus on gram-negative 
bacterial infections. J Intensive Care Med. 2017;32(1):25-37.

2. Sligl WI, Dragan T, Smith SW. Nosocomial gram-negative bacteremia in intensive care: epidemiology, antimicrobial susceptibilities, and outcomes. Int J Infect Dis. 2015;37:129-34.

3. Izadpanah M, Khalili H. Antibiotic regimens for treatment of infections due to multidrugresistant gram-negative pathogens: an evidencebased literature review. J Res Pharm Pract. 2015;4(3):105-14.

4. Spapen H, Jacobs R, Van Gorp V, Troubleyn J, Honoré PM. Renal and neurological side effects of colistin in critically ill patients. Ann Intensive Care. 2011;1(1):14. doi: 10.1186/2110-58201-14.

5. Falagas ME, Kasiakou SK. Toxicity of polymyxins: a systematic review of the evidence from old and recent studies. Crit Care. 2006; 10(1):R27. doi: 10.1186/cc3995.

6. Potron A, Poirel L, Nordmann P. Emerging broad-spectrum resistance in Pseudomonas aeruginosa and Acinetobacter baumannii: mechanisms and epidemiology. Int J Antimicrob Agents. 2015;45(6):568-85.

7. Qureshi ZA, Hittle LE, O'Hara JA, Rivera JI, Syed A, Shields RK, et al. Colistin-resistant Acinetobacter baumannii: beyond carbapenem resistance. Clin Infect Dis. 2015;60(9):1295-303.

8. Perry CM, Ibbotson T. Biapenem. Drugs 2002; 62(15):2221-34.

9. Jeon JH, Lee JH, Lee JJ, Park KS, Karim AM, Lee CR, et al. Structural basis for carbapenemhydrolyzing mechanisms of carbapenemases conferring antibiotic resistance. Int J Mol Sci 2015;16(5):9654-92.

10. Thamlikitkul V, Tiengrim S. In vitro activity of biapenem against gram-negative bacteria isolated from hospitalized patients at Siriraj Hospital. J Infect Dis Antimicrob Agents 2010; 27:55-9.

11. Chen H, Wang Z, Li H. In vitro analysis of activities of 16 antimicrobial agents against gram-negative bacteria from six teaching hospitals in China. Jpn J Infect Dis. 2015; 68(4):263-7.

12. Rajenderan S, Balaji V, Anandan S, Sahni RD, Tansarli GS, Falagas ME, et al. Determination of MIC distribution of arbekacin, cefminox, fosfomycin, biapenem and other antibiotics against gram-negative clinical isolates in South India: a prospective study. PLoS One 2014; 9(7):1-7.

13. Clinical and Laboratory Standards Institute. Performance Standards for Antimicrobial Susceptibility Testing; 26th Informational Supplement; 2016: M100-S25.

14. Magiorakos AP, Srinivasan A, Carey RB, Carmeli Y, Falagas ME, Giske CG, et al. Multidrug-resistant, extensively drug-resistant and pandrug-resistant bacteria: an international expert proposal for interim standard definitions for acquired resistance. Clin Microbiol Infect. 2012;18(3):268-81.

15. Teerawattanapong N, Panich P, Kulpokin D, Na Ranong S, Kongpakwattana K, Saksinanon A, et al. A systematic review of the burden of multidrug-resistant healthcare-associated infections among intensive care unit patients in Southeast Asia: the rise of multidrugresistant Acinetobacter baumannii. Infect Control Hosp Epidemiol. 2018;39(5):525-33.

16. Hsu LY, Apisarnthanarak A, Khan E, Suwantarat N, Ghafur A, Tambyah PA. Carbapenemresistant Acinetobacter baumannii and Enterobacteriaceae in South and Southeast Asia. Clin Microbiol Rev. 2017;30(1):1-22.

17. Christiansen KJ, Ip M, Ker HB, Mendoza M, Hsu L, Kiratisin P, et al. In vitro activity of doripenem and other carbapenems against contemporary gram-negative pathogens isolated from hospitalised patients in the Asia-Pacific region: results of the COMPACT Asia-Pacific study. Int J Antimicrob Agents 2010;36(6):501-6.

18. Kiratisin P, Chongthaleong A, Tan TY, et al. Comparative in vitro activity of carbapenems against major gram-negative pathogens: results of Asia-Pacific surveillance from the COMPACT II study. Int J Antimicrob Agents 2012; 39(4):311-6.

19. National Antimicrobial Resistance Surveillance Center (NARST). Antibiogram 2018: National Antimicrobial Resistance Surveillance Center, National Institute of Health, Department of Medical Sciences, Ministry of Public Health, Thailand; 2018 [cited 2019 May 1]. Available from:http://narst.dmsc.moph.go.th/antibiograms/ 2018/12/Jan-Dec2018-All.pdf. 
20. National Antimicrobial Resistance Surveillance Center (NARST). Result of antimicrobial resistance surveillance: National Antimicrobial Resistance Surveillance Center, National Institute of Health, Department of Medical Sciences, Ministry of Public Health, Thailand; 2018 [cited 2019 May 1]. Available from: http://narst.dmsc.moph.go.th/data/AMR $\% 20$ 2000-2018-12M.pdf.

21. An JH, Kim YH, Moon JE, Jeong JH, Kim $\mathrm{SH}$, Kang SJ, et al. Active surveillance for carbapenem-resistant Acinetobacter baumannii in a medical intensive care unit: can it predict and reduce subsequent infections and the use of colistin?. Am J Infect Control. 2017;45(6): 667-72.

22. Liu WL, Liang HW, Lee MF, Lin HL, Lin $\mathrm{YH}, \mathrm{Chen} \mathrm{CC}$, et al. The impact of inadequate terminal disinfection on an outbreak of imipenem-resistant Acinetobacter baumannii in an intensive care unit. PloS One. 2014;9(9): e107975.doi:10.1371/journal.pone.0107975.
23. Hang Y, Chen Y, Xue L, Sun S, Liu L, Gao J, et al. Evaluating biapenem dosage regimens in intensive care unit patients with Pseudomonas aeruginosa infections: a pharmacokinetic/ pharmacodynamic analysis using Monte Carlo simulation. Int J Antimicrob Agents. 2018;51 (3):484-87.

24. Leelasupasri S, Santimaleeworagun W, Jitwasinkul T. Antimicrobial susceptibility among colistin, sulbactam, and fosfomycin and a synergism study of colistin in combination with sulbactam or fosfomycin against clinical isolates of carbapenem-resistant Acinetobacter baumannii. J Pathog. 2018; 3893492. doi: 10.1155/2018/3893492.

25. Singkham-In U, Chatsuwan T. In vitro activities of carbapenems in combination with amikacin, colistin, or fosfomycin against carbapenemresistant Acinetobacter baumannii clinical isolates. Diagn Microbiol Infect Dis. 2018; 91(2):169-74. 